\title{
Role of Hybrid LEVEL SET IN FETAL CONTOUR EXTRACTION
}

\author{
Rachana Jaiswal and Srikant Satarkar \\ Department of Computer Science \& Engineering, Amravati University, Akola, MH
}

\begin{abstract}
Image processing technologies may be employed for quicker and accurate diagnosis in analysis and feature extraction of medical images. Here, existing level set algorithm is modified and it is employed for extracting contour of fetus in an image. In traditional approach, fetal parameters are extracted manually from ultrasound images. An automatic technique is highly desirable to obtain fetal biometric measurements due to some problems in traditional approach such as lack of consistency and accuracy. The proposed approach utilizes global \& local region information for fetal contour extraction from ultrasonic images. The main goal of this research is to develop a new methodology to aid the analysis and feature extraction.
\end{abstract}

\section{KEYWORDS}

Active contour, Global Region-based, Local Region-based, Edge-based

\section{INTRODUCTION}

Image segmentation permits visualization of organs and structures in the body, analysis of the shapes and sizes of the organs, and diagnosis of pathologies more quickly and accurately. High variations from one expert to another exists in physical segmentation due to its dependence on physician's subjective knowledge and his experience. Thus, it is a tiresome, time consuming process and most of the times it is not accurate. The information generated from the computerized analysis of medical images can be used as a second opinion in making diagnostic decisions by physicians.

Due to presence of inhomogeneous intensity profile, strong speckle noise and shadows in fetal images, it becomes difficult to properly segment the correct positions and shapes of interesting regions. Also, in fetal images, edges are poorly defined and are close to other fetal structures complicating segmentation task further. The proposed approach aims at developing a novel segmentation technique which will extract fetal contour accurately with reduced dependence on initial curve placement [9].

\section{IMAge SEgmentation TeChNiQue}

Segmentation algorithms can be classified into similarity-based and discontinuity-based approach. Image is partitioned based on uniformity in intensity and texture in similarity-based approach whereas discontinuity-based approach partitions image based on sudden intensity changes. Similarity-based methods create more coherent regions compared to discontinuity-based methods. In this approach, segmentation is performed without taking account of connectivity property. It is based on the grey value of pixels. In images, object boundaries represent edges which occur at the point of intersection of two regions where intensities vary. Sobel, Robert and 
Signal \& Image Processing: An International Journal (SIPIJ) Vol.12, No.1, February 2021

Prewitt operators were used for edge detection in traditional approach. Another discontinuitybased approach involves use of deformable model.



Figure 1: Image Segmentation Algorithm classification

Closed parametric curves or surfaces from images are generated by deformable model which are robust to noise and spurious edges [2]. Depending on way of evolution, deformable models can be categorized into the parametric deformable models and the geometric model. Parametric model uses Lagrange formulation. It is initialization sensitive. It involves less human intervention. Geometric model evolves according to the Euler formulation. Initialization sensitivity of geometric model is less compared to parametric ones. It can detect multiple boundaries at a time. It reduces the time required for image analysis and subjectivity associated with manual delineations and measurements [6].

\section{IMAGE SEgmentation BASED ON LEVEL SET}

Stanley Osher and James Sethian proposed level set approach. Level set approach is employed in medical, satellite and natural image segmentation, analysis and boundary extraction. It is capable of handling corner, inflexion and topological structure changes and can also segment regions with irregular shape. It is insensitive to initialization [3]. It is capable of segmenting objects with deep concavities and detecting multiple objects [4].

For contour $\mathrm{C}$ evolving by the time $\mathrm{t}$ in the normal vector direction $\mathrm{N}$ with the speed function $\mathrm{F}$ (K) which depend on the curvature of the contour, contour deformation using PDE can be written as,

$$
\frac{\partial c}{\partial t}=F(k) \cdot N
$$


The contour propagation speed is higher in a high curvature area compared to a low curvature area [8]. Minimization of internal and external energies along the boundary of curve is achieved by deforming the curve along its shape [10].

A signed distance function (SDF) is given by $\phi(x, y)$ for a closed curve $\mathrm{C}$ which divides plane into two regions. $\phi(x, y)>0$ for point lying inside the curve $\mathrm{C}$; for outside point, $\phi(x, y)<0 ; \phi$ $(x, y)=0$ for the point lying on $\mathrm{C}$ [7]. Topological changes can be handled naturally using following equation:

$$
\frac{\partial \emptyset}{\partial t}+F(K) \cdot|\nabla \emptyset|=0
$$

Level set is not capable of detecting objects in images with low-contrast boundaries [5]. Traditional level set method suffers from leaking problem in presence of a fuzzy or discrete boundary in the region. Also, too large or too small initial contour [11] affects the iteration time. Traditional approach can be further classified into edge-based and region-based models. Edgebased approach relies on local edge information for curve evolution whereas region-based approach utilizes statistical information.

Edge-based approach uses image gradient as edge stopping function. It is highly sensitive to noise, affected by fuzzy or blurred boundaries. Affected by initial curve placement. Uses local edge information Can segment heterogeneous objects Have high segmentation precision since it allows user to mark boundary directly. In Region-based approach, the motion of the contour relies on region descriptor. It is insensitive to noise and is less affected by fuzzy or blurred boundaries. It can detect interior and exterior boundaries simultaneously. It is robust to initial curve placement and is not ideal for segmenting heterogeneous objects.

Geometric active contour models can be distinguished as (a) Geodesic Active Contour Model; (b) Mumford-Shah Model; (c) Chan-Vese Model [6].

\subsection{Geodesic Active Contour (GAC) Model}

It is a simplified snake model without second order smoothness. It has features from both level set and snake algorithm. In geodesic active contour, energy expression comprises of attraction component and regularity component. It utilizes the image gradient to derive the edge stopping function (ESF) for locating the contour around the boundaries of desired object. It obtains object boundary using minimal weighted length curve. Let $\Omega$ be a bounded open subset of $\mathrm{R}^{2}, I: \Omega \rightarrow R$ is an input image and $C(q)$ is a closed curve. The energy functional for GAC model is given by following equation:

$$
\operatorname{EGAC}(C(q))=\int_{0}^{1} g(\nabla I(C(q)) \mid) C^{\prime}(q) d q
$$

It enabled extraction of smooth contours. It can detect several objects simultaneously. It cannot retrieve angles. It fails to segment discrete shape boundary target and objects with weak boundary. It is difficult for the GAC model to find the target when the initial contour is far away from the target boundary. It falls into local minimum when the initial contour is not placed near object boundaries. 
Signal \& Image Processing: An International Journal (SIPIJ) Vol.12, No.1, February 2021

\subsection{Chan-Vese Model}

Edge-based active contour models suffer from boundary leakage problems because of the presence of image noise, weak contrast and intensity inhomogeneity, which generally occurs in a variety of medical images with varying contrast. In order to avoid limitations of edge-based models, Chan and Vase introduced the first region-based active contour model (CV).

It is a non-parametric, minimal partition problem based on an energy minimization of MumfordShah technique to extract the boundaries not necessarily detected using the image gradient. It is based on homogeneity criteria of both the object to be segmented and background.

Let I be the given image in the domain $\Omega$ with $\mathrm{c} 1$ and $\mathrm{c} 2$ the mean intensities inside and outside the contour respectively. The CV model is formulated by minimizing following energy functional.

$$
\begin{aligned}
& E(C, C 1, C 2)=\mu \text { Length }(C)+v \cdot \text { Area }(\text { inside }(C)) \\
& \quad+\lambda 1 \int_{\text {inside }(C)}|I(x, y)-c 1|^{2} d x d y+\lambda 2 \int_{\text {outside }(C)}|I(x, y)-c 2|^{2} d x d y
\end{aligned}
$$

where $\mu, \lambda_{1}, \lambda_{2}>0 . \lambda_{1}, \lambda_{2}=1$. The first two terms help the curve to be regular, and the two others correspond to the energy inside and outside the curve. Regularization term ensures that level set function is close to signed distance function. The minimum of the previous energy is obtained when the curve is on the boundaries of the object to be detected [3]. The third and fourth term are the region fitting terms, which drives the contour to the object boundaries during curve evolution.

The first term in above equation, represents the edge contours length for a given segmentation. If we expect a region with a smooth boundary, we might weight this term more heavily to avoid finding a complex (and therefore long) perimeter. Penalty on the total area of the foreground region found by the segmentation is second term.

The third term, represents the variance of the image gray level in the foreground region which measures how uniform the region is in terms of pixel intensity. Similarly, the fourth term measures for the background region. Minimizing the sum of these last two terms, leads to a segmentation into a foreground and background region that are each as uniform as possible.

$$
\frac{\partial \phi}{\partial \mathrm{t}}=\partial(\phi)\left[\mu \operatorname{div}\left(\frac{\nabla \phi}{|\nabla \phi|}\right)-\lambda_{1}\left(\mathrm{I}-\mathrm{c}_{1}\right)^{2}+\lambda_{2}\left(\mathrm{I}-\mathrm{c}_{2}\right)^{2}-\mathrm{v}\right]
$$

The zero-level set function represents the initial contour curve. Updating the level set function by above formula, when the iteration process has finished, we can get the final contour curve through the zero-level set function.

$\mu$ is used for controlling the smoothness of zero level set, $v$ - increases propagation speed.

The CV model can automatically detect all the contours, no matter where the initial contour starts in the image. So, we can say that the CV model has the global segmentation property to segment all objects in an image. Comparatively, the GAC model can only extract the object when the initial contour surrounds its boundary, and it can-not detect the interior contour without setting 
Signal \& Image Processing: An International Journal (SIPIJ) Vol.12, No.1, February 2021

the initial one inside the object. Thus, we can say that the GAC model possesses local segmentation property which can only segment the desired object with a proper initial contour.

$\mathrm{C}-\mathrm{V}$ model can segment images having close convex contours and comparative clear boundaries. $\mathrm{C}-\mathrm{V}$ model can solve problem of fuzzy boundaries. C-V model loses the capability of identifying the foreground and background regions when $\mathrm{c} 1$ is approximately equal to $\mathrm{c} 2$, which means that image intensities are severely inhomogeneous.

\section{LEVEL SET IMAGE SEGMENTATION IMPROVEMENT}

\subsection{Proposed Global Region-based Model}

The proposed model is based on active contours which evolve in time according to geometric measures of the image for the detection of object boundaries. I used existing preprocessing method in order to reduce effect of noise and to preserve the sharpness of edges. An anisotropic diffusion is applied prior to segmentation. Stability of the curve evolution is destroyed because of irregularities developed by the level set function (LSF) during its evolution. A re-initialization procedure is used to restore the irregularity of the

LSF and maintain stable level set evolution process. Re-initialization procedure helps in keeping the evolving LSF close to signed distance function (SDF). The proposed method is robust to initial curve placement. The performance of proposed model is better in the presence of image noise and weak object boundaries. This method relies on constant intensity information across the regions therefore, it fails to segment images with intensity inhomogeneity.

\subsection{Proposed Local Region-based Model}

Medical images possess inhomogeneous gray intensity which gives erroneous results. So, in order to handle gray intensity inhomogeneity, local region-based approach is proposed. An energy function based on local image statics capable of segmenting objects with heterogeneous feature profiles is formulated. In proposed approach, for sound curve evolution and reliable results, re-initialization is used. To segment images with intensity inhomogeneity, proposed method considers the local image information energy. Foreground and background are modeled as constant intensities using $\mathrm{u}_{\mathrm{X}}$ and $\mathrm{v}_{\mathrm{X}}$ by replacing global means with local equivalents. The points inside and outside the object are modeled using the mean intensities of the local regions at each point on the true edge of an object. The resulting flow is more robust to image noise, capable of finding significant local minima and partitioning the image without making global assumptions about its makeup. In proposed approach, small local regions represent target and background. The proposed algorithm drives the evolving curve according to energy function in a small local area. The framework utilizes local region information in active contour evolution rather than global statistical information.

$$
\begin{gathered}
\mathrm{V}(\mathrm{x}, \mathrm{y})=1,\|\mathrm{x}-\mathrm{y}\| \\
\quad=0 \text { length of side of square window } \\
=0 \text { otherwise }
\end{gathered}
$$

$\mathrm{V}(\mathrm{x}, \mathrm{y})$ represents local mask. It is used to mark the local region of each pixel $\mathrm{x}$ along the curve. The function denotes the judgement value. When point $\mathrm{y}$ is inside the square with center at $\mathrm{x}$, the value is 1 . Otherwise, the value is 0 . For Heaviside function $\mathrm{H} \phi(\mathrm{y})$, the intensity distribution inside the curve can be represented by $\mathrm{V}(\mathrm{x}, \mathrm{y}) \cdot \mathrm{H}(\phi(\mathrm{x}, \mathrm{y}))$ and intensity distribution outside the curve is given by $\mathrm{V}(\mathrm{x}, \mathrm{y}) \cdot(1-\mathrm{H}(\phi(\mathrm{x}, \mathrm{y}))) \cdot \mathrm{u}_{x}$ and $\mathrm{v}_{x}$ are mean intensities of internal and external region respectively. The energy is given by 
Signal \& Image Processing: An International Journal (SIPIJ) Vol.12, No.1, February 2021

$$
\begin{aligned}
\mathrm{E}(\phi)=\int_{\Omega_{\mathrm{x}}} \delta \phi & (\mathrm{x}) \int_{\Omega_{\mathrm{y}}} \mathrm{V}(\mathrm{x}, \mathrm{y})\left[\mathrm{H}(\phi(\mathrm{y}))\left(\mathrm{I}(\mathrm{y})-\mathrm{u}_{\mathrm{x}}\right)^{2}\right. \\
& \left.+(1-\mathrm{H}(\phi(\mathrm{y})))\left(\mathrm{I}(\mathrm{y})-\mathrm{v}_{\mathrm{x}}\right)^{2}\right] d \mathrm{ydx} \\
& +\lambda \int_{\Omega_{\mathrm{x}}} \delta \phi(\mathrm{x})|| \nabla \phi(\mathrm{x})|| \mathrm{dx}
\end{aligned}
$$

The proposed model is able to handle noisy, blurry boundaries object, objects with heterogeneous feature profiles.

Motivated by the work of [15], a hybrid segmentation approach which aims to blend the benefits of global and local region-based approaches to improve the quality of segmentation is proposed. It is a two-stage method which uses local and global intensity information. A rough segmentation result is obtained using two stage global region-based approach. It is fast and stable with homogeneous regions. This result is given as input to second stage to obtain final segmentation result. Desirable segmentation result is achieved using second stage. The experimentation was carried out on ultrasound images obtained from authorized radiologist.

MATLAB is used for experimentation on personal computer with Intel Core i5, $1.7 \mathrm{GHz}$ and 4GB RAM. Sufficient medical images are tested and after testing, result shows improvement in quality and efficiency of segmentation as compared to existing approaches. The values of Structural Similarity Index, Correlation, Mean Square Error, Peak Signal to Noise Ratio, Dice similarity coefficient, Jaccard Similarity Coefficient are evaluated for different images using various algorithms. The images are taken from authorized sonography center. The result of the evaluated values of Structural Similarity Index, Correlation, Mean Square Error, Peak Signal to Noise Ratio, Dice similarity coefficient, Jaccard Similarity Coefficient for some of the images are represented in comparison graph. Proposed approach when tested over a large dataset of fetal images has given encouraging results. The results which are presented shows Structural Similarity index for different images using proposed approach performs $6.94 \%$ better over existing method, for Correlation it is 5.97\%, MSE is decreased by $66.06 \%$, Peak Signal to Noise ratio is improved by $16.73 \%$, for Dice Similarity Coefficient it is $5.9 \%$ and for Jaccard Similarity Coefficient it is $29.89 \%$. So, it is proposed after careful observations that the hybrid approach gives better performance among all other segmentation techniques.

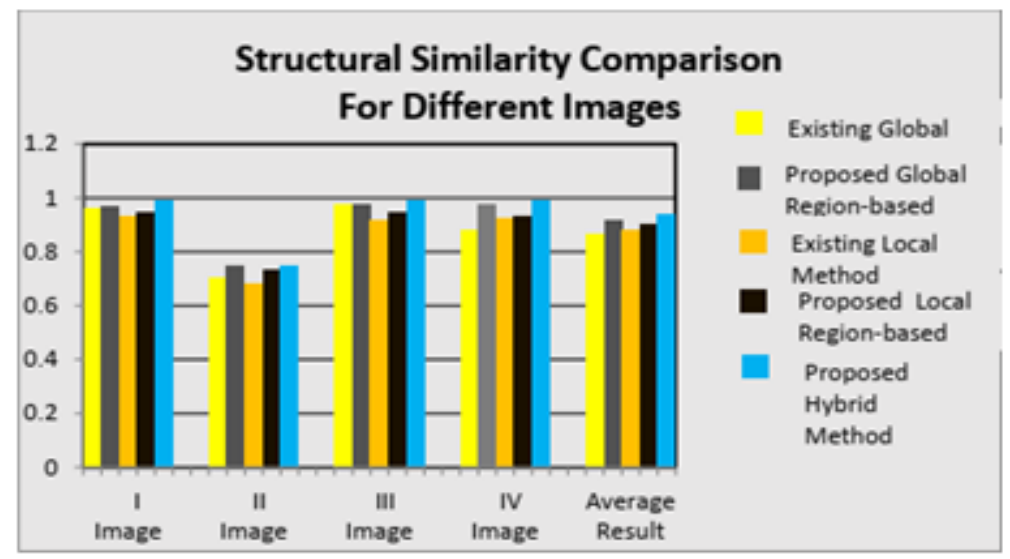

Figure 2: Structural Similarity Comparison for different images 
Signal \& Image Processing: An International Journal (SIPIJ) Vol.12, No.1, February 2021



Figure 3: MSE Comparison for different images



Figure 4: Correlation Comparison for different images

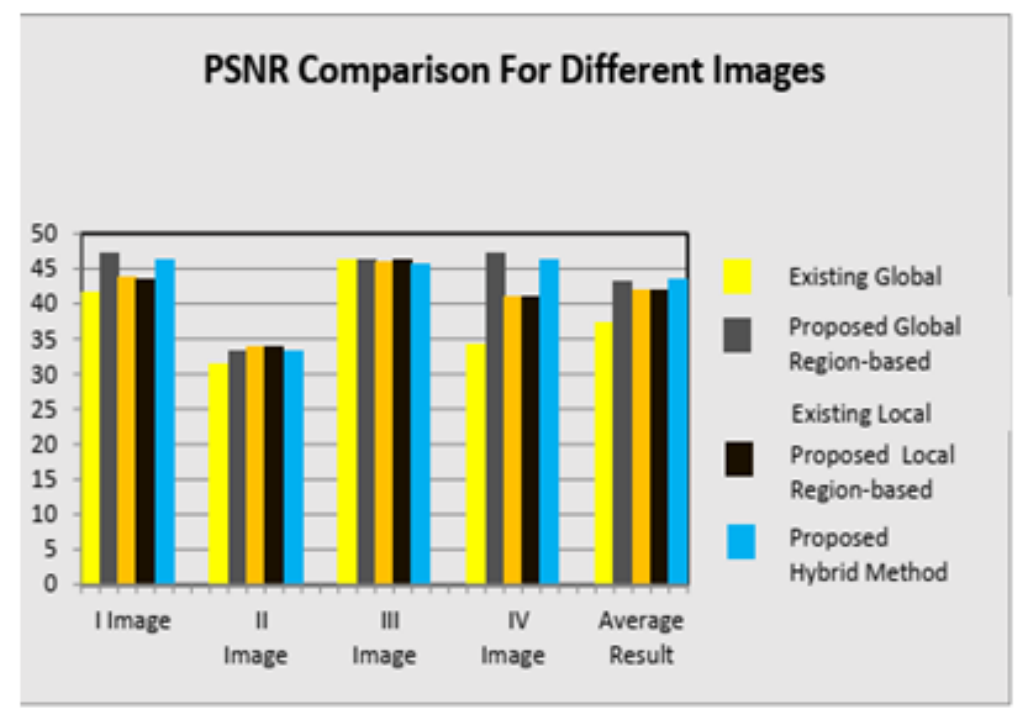

Figure 5: Peak Signal to Noise Ratio Comparison for different images 
Signal \& Image Processing: An International Journal (SIPIJ) Vol.12, No.1, February 2021

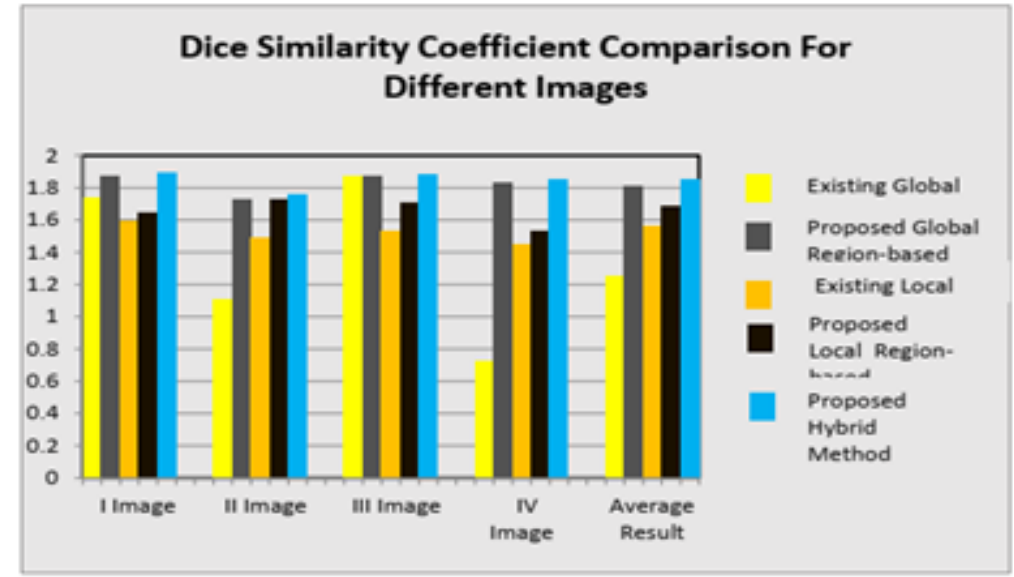

Figure 6: Dice Similarity Coefficient Comparison for different images

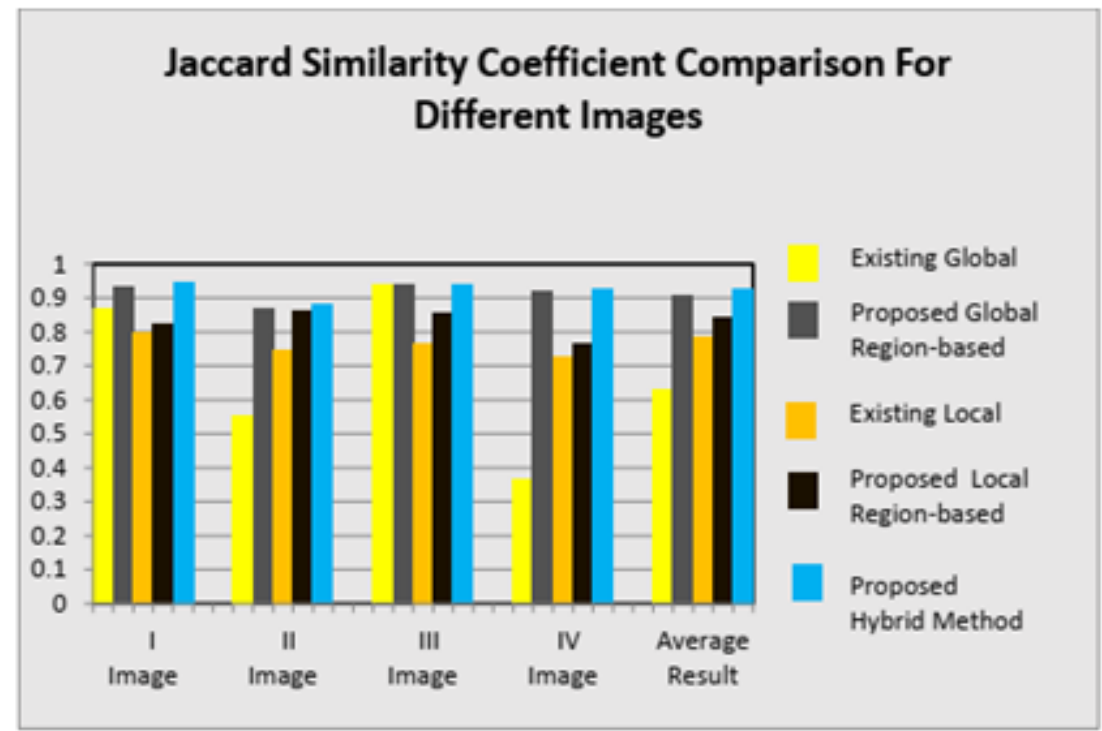

Figure 7: Jaccard Similarity Coefficient Comparison for different images

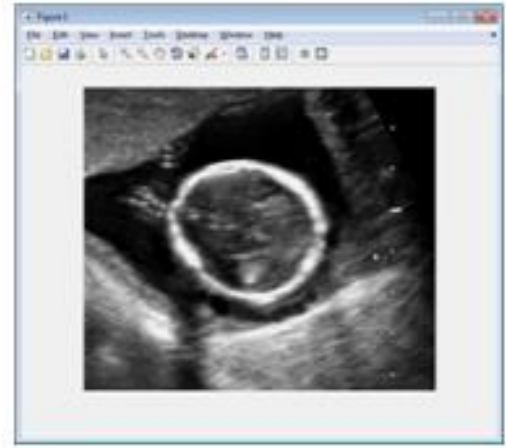

Figure 8: a) Input image

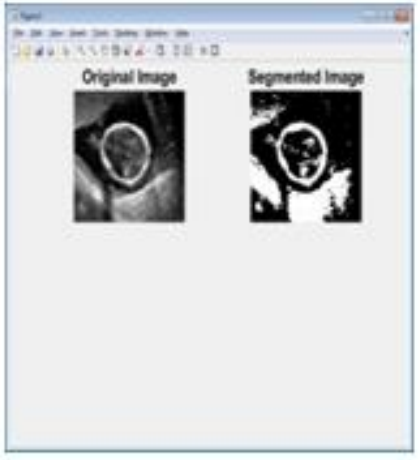

b) Result of thresholding

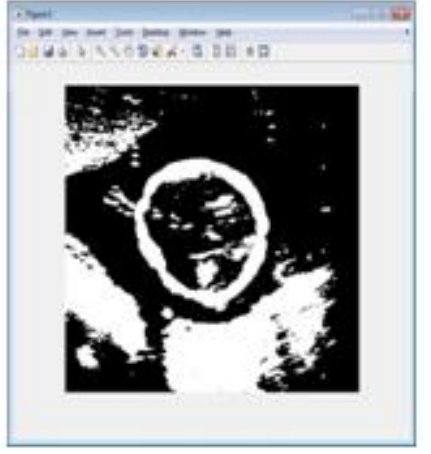

c) Result of K-Means 
Signal \& Image Processing: An International Journal (SIPIJ) Vol.12, No.1, February 2021

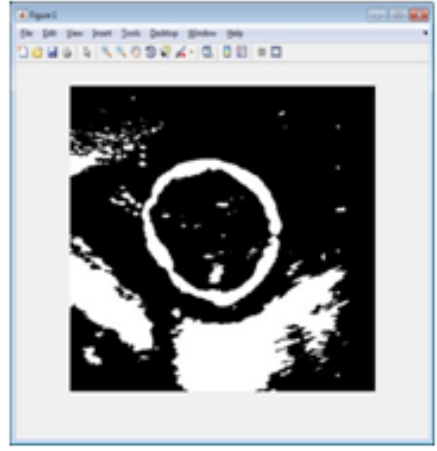

d) Region Growing

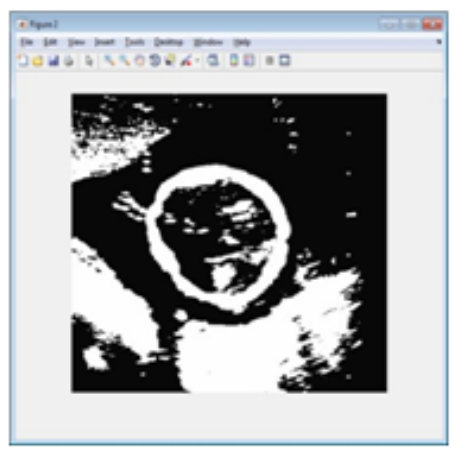

e) Fuzzy C-Means



Figure 9 a) Input image

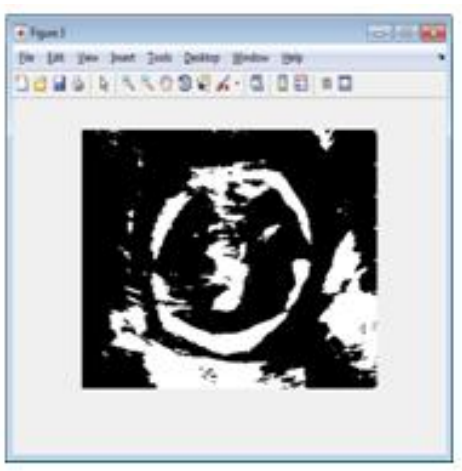

b) Fuzzy C-Means

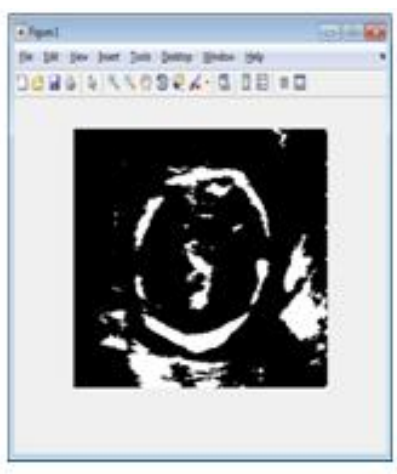

c) Region Growing

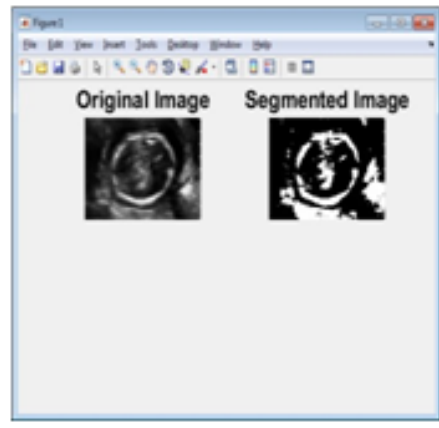

d) Thresholding



e) K-Means

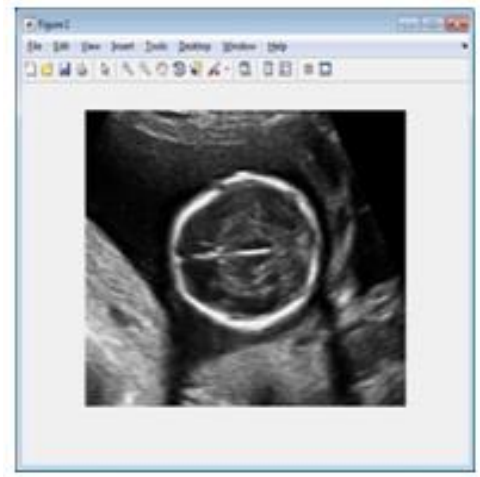

Figure 10: a) Input Image



b) Fuzzy C-Means

c) Region Growing 
Signal \& Image Processing: An International Journal (SIPIJ) Vol.12, No.1, February 2021

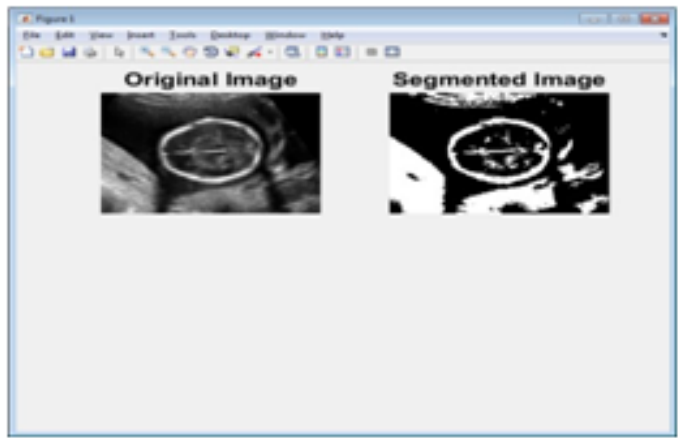

d)Thresholding

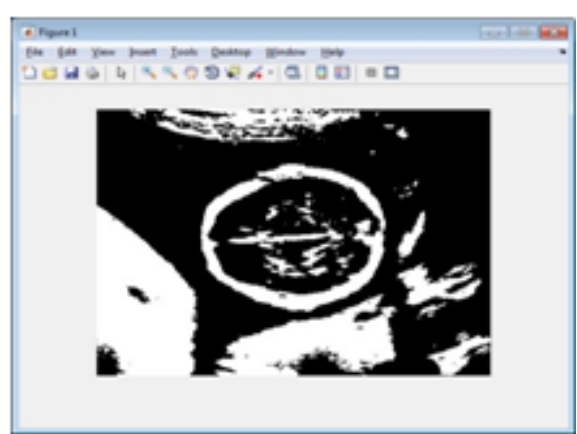

e) K-Means

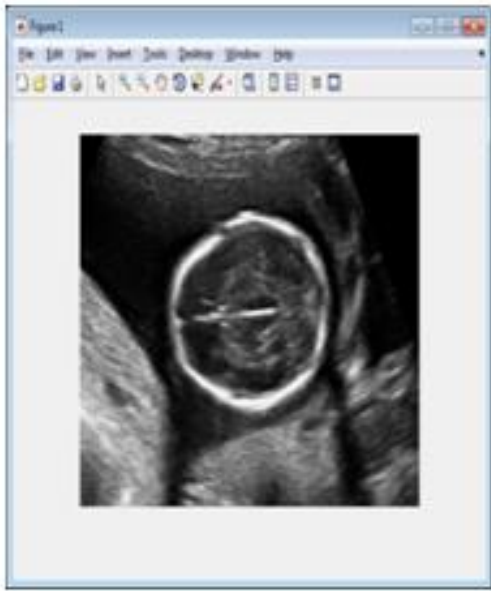

Figure 11: a) Input Image

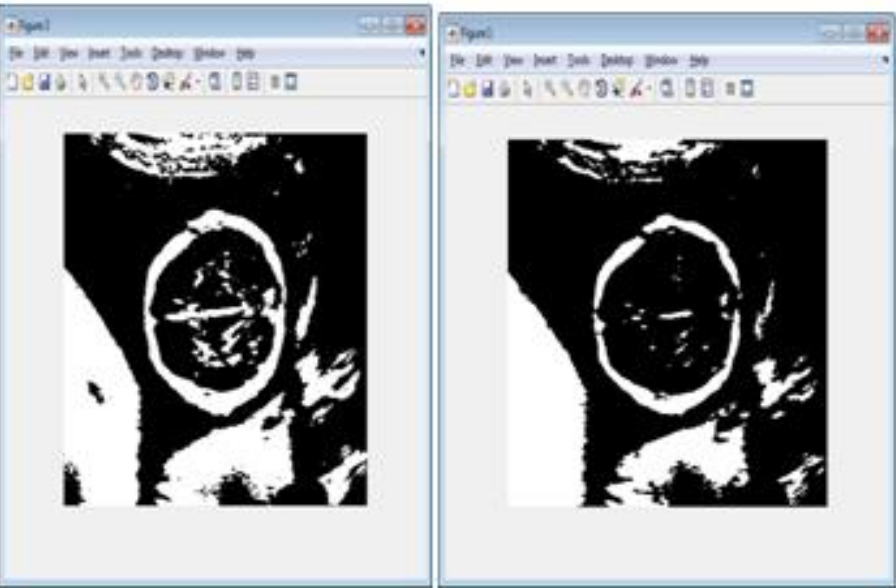

b) Fuzzy C-Means

c) Region Growing

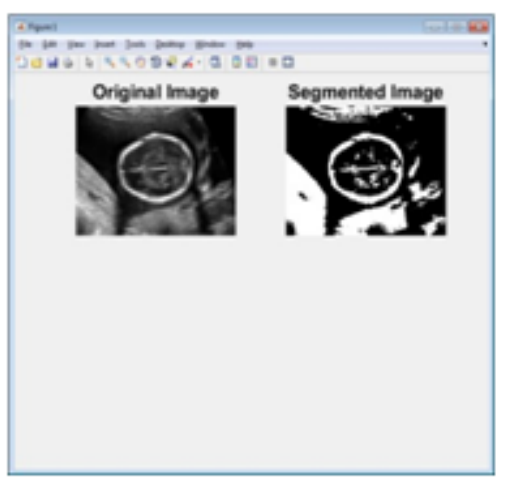

d)Thresholding

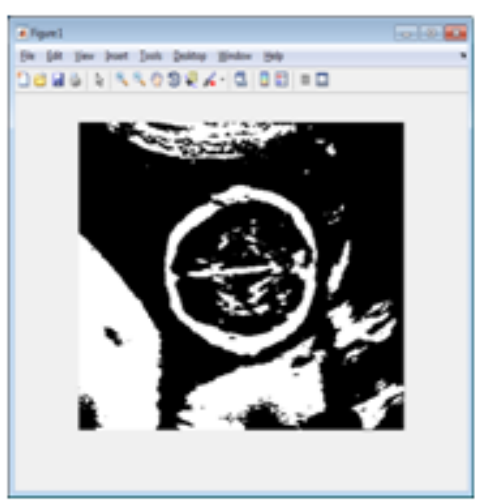

e) K-Means 
Signal \& Image Processing: An International Journal (SIPIJ) Vol.12, No.1, February 2021

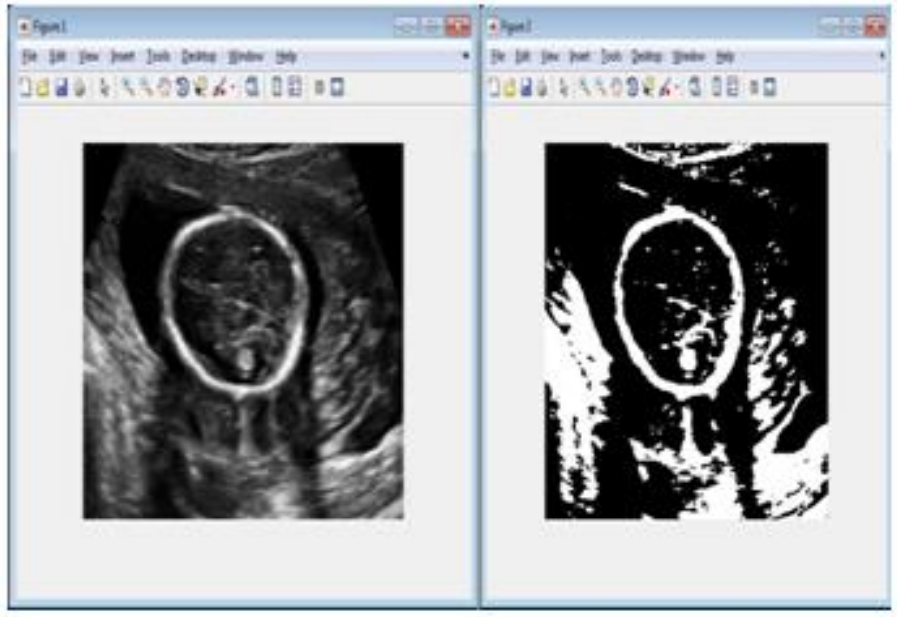

Figure 12: a) Input image

b) Fuzzy C-Means

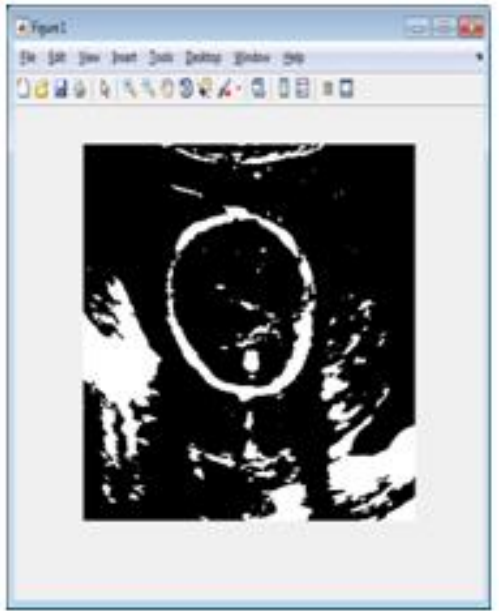

c) Region growing

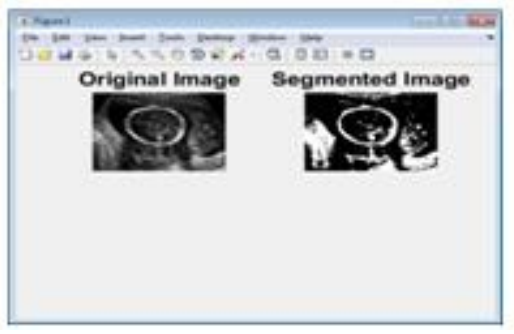

d) Thresholding

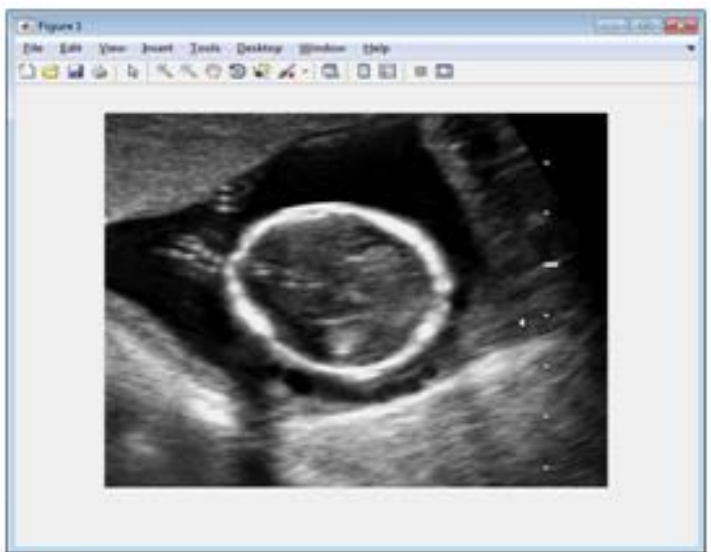

Figure 13: a) Input Image

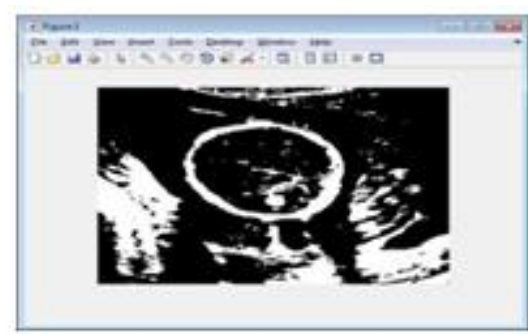

b) K-Means

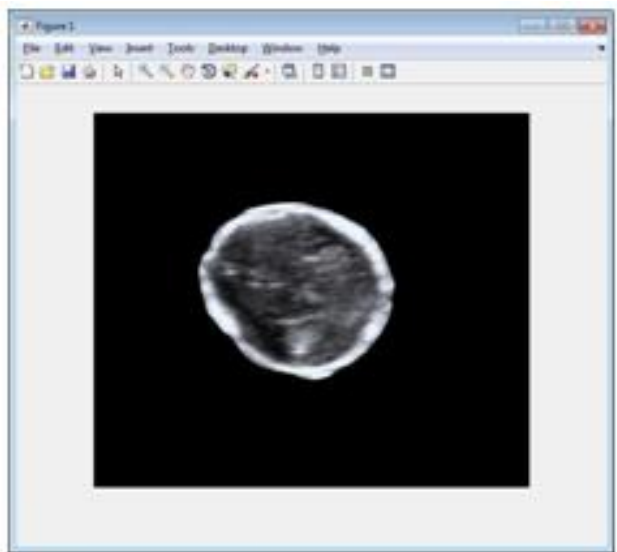

b) Output using proposed approach 
Signal \& Image Processing: An International Journal (SIPIJ) Vol.12, No.1, February 2021

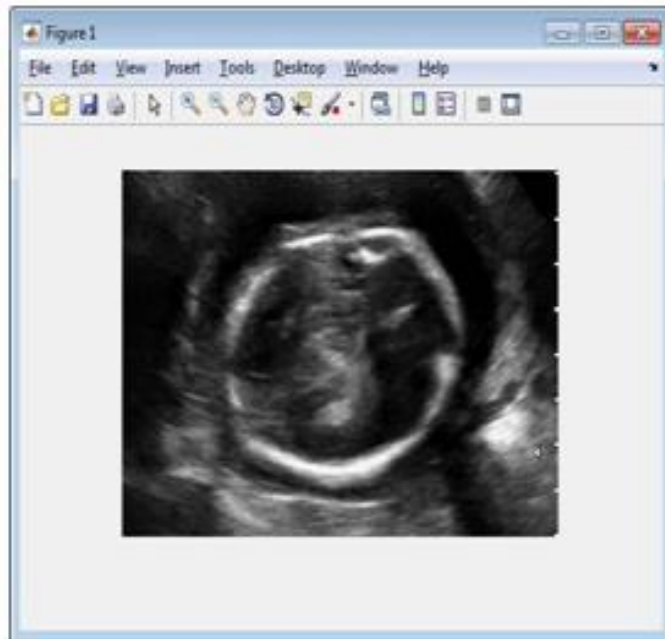

Figure 14: a) Input Image

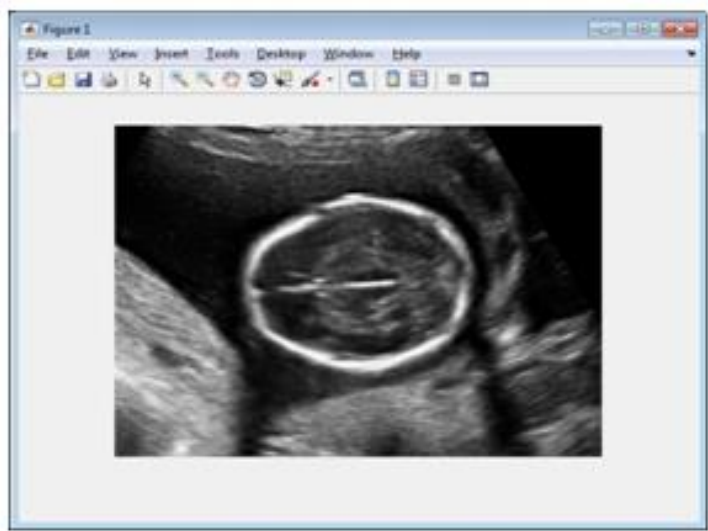

Figure 15: a) Input Image

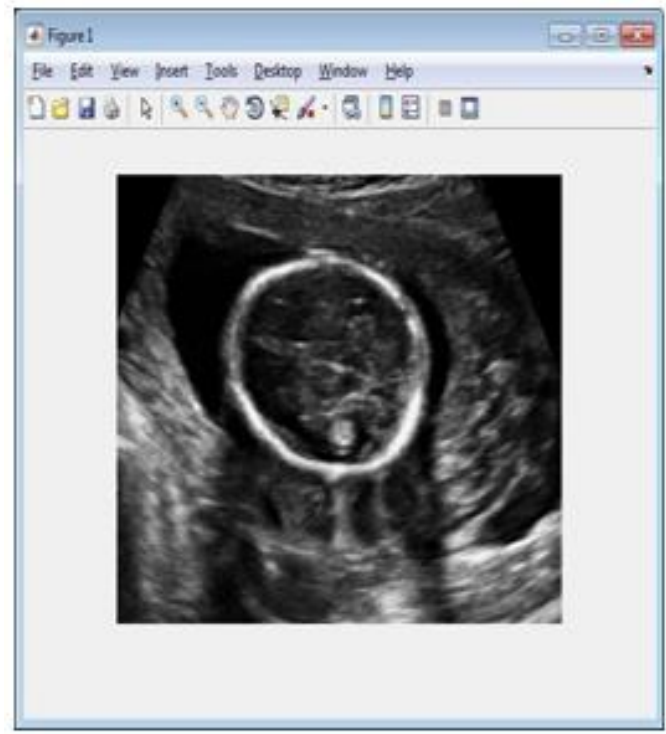

Figure 16: a) Input Image



b) Output using proposed approach

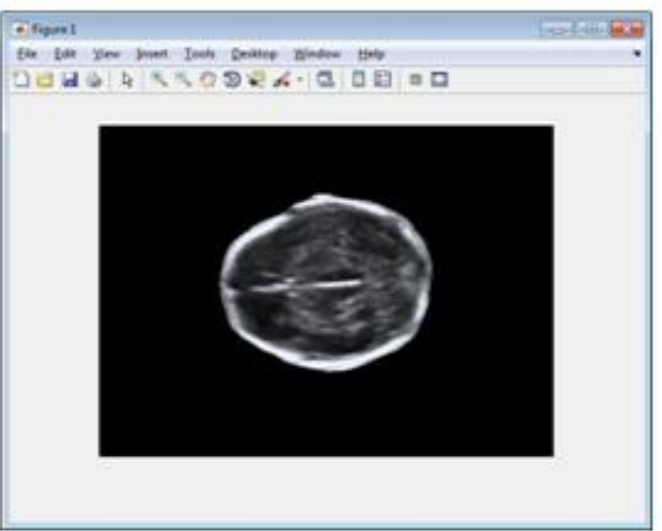

b) Output using proposed approach



b) Output using proposed approach 


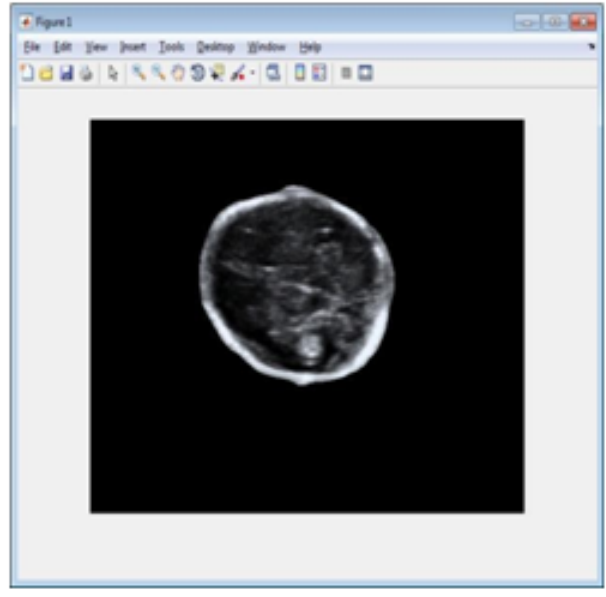

Figure 17: a) Input Image

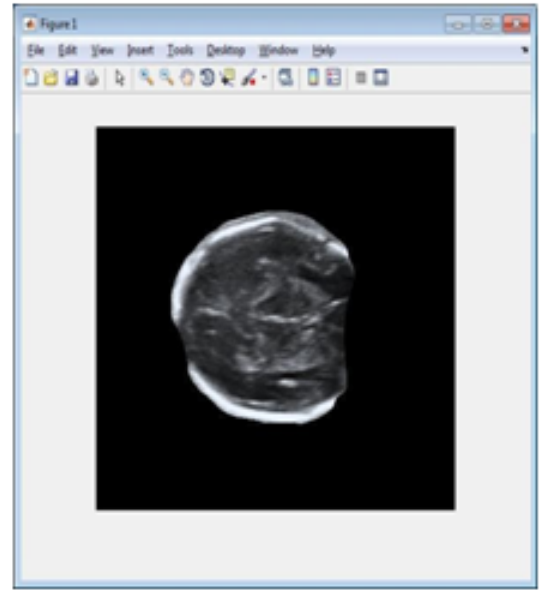

b) Output using proposed approach

\section{CONClusion}

Perona and Malik smoothing technique is applied prior to segmentation [16]. The narrow band method is employed in the proposed approach. The proposed global region- based model is insensitive to the location of initial contour. The model could not produce correct result in presence of heterogeneous object. So, after observing the performance of global region-based approach, local region-based approach is implemented. It is a local method, so in presence of heterogeneous object or incomplete edges, this algorithm produces correct result. In order to obtain benefits of both methods, result after application of proposed global method which is rough segmentation is given as input to proposed local method for suitable initialization. The segmentation method is robust to different types of initialization and provides more accurate results.

\section{REFERENCES}

[1] [Wang et. al., 2004] Zhou Wang, Alan C. Bovik, Hamid R. Sheikh and Eero P. Simoncelli, (2004) "Image quality assessment: From error visibility to structural similarity," IEEE Transactions on Image Processing, pp.600-612.

[2] [Wang et.al., 2010] HaiJun Wang, Ming Liu and WenLai Ma, (2010) "Color Image Segmentation Based on a New Geometric Active Contour Model", International Conference on Machine Vision and Human-machine Interface, DOI: 10.1109/MVHI.2010.75, pp. 6 -9.

[3] [Charmi et.al., 2010] Mohamed Ali Charmi, Mohamed Amine Mezghich, Slim M'Hiria, Stephane Derrode and Faouzi Ghorbel, (2010) "Geometric shape prior to region-based active contours using Fourier-based shape alignment", IEEE International Conference on Imaging Systems and Techniques, DOI: 10.1109/IST.2010.5548489, pp.478 - 481, 2010.

[4] [Chiu et. al., 2010] Yun-Jen Chiu, Van-Truong Pham, Thi-Thao Tran and Kuo-Kai Shyu,(2010) "Evaluation of active contour on medical inhomogeneous image segmentation", 3rd International Conference on Computer Science and Information Technology, DOI:10.1109/ICCSIT.2010.5564101,pp.311 - 314, 2010.

[5] [Ebrahimdoost et.al., 2010] Y.Ebrahimdoost, J.Dehmeshki, T.S.Ellis, M.Firoozbakht, A.Youannic and SD.Qanadli,(2010) "Medical Image Segmentation Using Active Contours and a Level Set Model: Application to Pulmonary Embolism (PE) Segmentation", IEEE Xplore, DOI: 10.1109/ICDS.2010.64, pp.269-273, 2010.

[6] [Cheng et. al.,2011] Jieyu Cheng, Mingyue Ding and Xuming Zhang,(2011) "Geometric Active Contour Model and its Application to Carotid Plaque Detection", International Conference on Intelligent Computation and Bio-Medical Instrumentation, DOI:10.1109/ICBMI.2011.62, pp.101 $104,2011$. 
Signal \& Image Processing: An International Journal (SIPIJ) Vol.12, No.1, February 2021

[7] [Fan et. al.,2012] Jinping Fan, Ruichun Wang, Shiguo Li and Chunxiao Zhang, (2012) "Automated cervical cell image segmentation using level set based active contour model", in 12th International Conference on Control Automation Robotics \& Vision (ICARCV),DOI: 10.1109/ICARCV.2012.6485273, pp.877 - 882, 2012.

[8] [Goceri et. al., 2012] Evgin Goceri, Mehmet Z. Unlu, Cuneyt Guzelis and Oguz Dicle, (2012) “An automatic level set based liver segmentation from MRI data sets", $3^{\text {rd }}$ International Conference on Image Processing Theory, Tools and Applications (IPTA), DOI: 10.1109/IPTA.2012.6469551, pp.192 - 197, 2012.

[9] [Li-Zhi et.al., 2012] Xie Li-Zhi, Tian Yun, Zhou Ming-Quan and Cao Rong-Fei, (2012) "Cerebrovascular segmentation based on region growing and level set algorithm", International Conference on Audio, Language and Image Processing, DOI:10.1109/ICALIP.2012.6376733, pp. $854-859,2012$.

[10] [Mouhadjer et.al.,2013] Hassan Mouhadjer, M. Mansour, M. Ouslim and B. Bouchiba, (2013) "Segmentation of human chromosome images using distance regularized level set evolution", 2nd International Conference on Advances in Biomedical Engineering, DOI:10.1109/ICABME.2013.6648 886, pp.215 - 218, 2013.

[11] [Chen et.al.,2014] Mengjuan Chen, Jianwei Li, Hanqing Zhao and Xiao Ma, (2014) “A local region-based level set algorithm for image segmentation", IEEE Workshop on Electronics, Computer and Applications, DOI: 10.1109/IWECA.2014.6845753, pp.844 - 847, 2014.

[12] [Liu et.al.,2015] Zhiwei Liu, Dongao Zhou, Qiang Lin and Jiayu Lin, (2015) “Active Contour Model Based on Local and Global Image Information", 7th International Conference on Intelligent HumanMachine Systems and Cybernetics, DOI: 10.1109/IHMSC.2015.63, pp.266 - 269, 2015.

[13] [Kulkarni and Shitole, 2016] Snehal Kulkarni and Sanjay Shitole, (2016) "Region growing based novel approach for estimation of speckle in SAR images", IEEE Bombay Section Symposium (IBSS), DOI: 10.1109/IBSS.2016. 7940 200, pp.1-5, 2016.

[14] [Saini and Sahni, 2017] Harmeet Saini and Varsha Sahni, (2017) "Region Growing segmentation using de-noising algorithm for medical ultrasound images", 3rd International Conference on Computational Intelligence \& Communication Technology (CICT), DOI: 10.1109/CIACT.2017 .7977 313, pp. $1-5,2017$.

[15] [Soomro et. al., 2018] Soomro S, Munir A and Choi KN, "Hybrid two-stage active contour method with region and edge information for intensity inhomogeneous image segmentation", PLOS ONE 13(1): e0191827, [Online]. Available: https://doi.org/10.1371/ journal.pone. 0191827.

[16] [Soomro and Choi,2018] Shafiullah Soomro and Kwang Nam Choi, (2018) "Active Contours Based on An Anisotropic Diffusion", Digital Image Computing: Techniques and Applications (DICTA), DOI: 10.1109/DICTA.2018.8615767, pp. 1 - 6, 2018 